\title{
Microdream neurophenomenology
}

\section{Tore Nielsen*}

\author{
Dream \& Nightmare Laboratory, Center for Advanced Research in Sleep Medicine, Hopital du Sacre-Coeur de \\ Montreal and Department of Psychiatry, University of Montreal, Canada
}

*Correspondence address. Center for Advanced Research in Sleep Medicine, CIUSSS-NIM - Hopital du Sacre-Coeur de Montreal, 5400 Boul. Gouin Ouest, Montreal, Quebec, H4J 1C5 Canada. Tel: +514-338-2222x3350; Fax: +514-338-2693; E-mail: Tore.nielsen@umontreal.ca

\begin{abstract}
Nightly transitions into sleep are usually uneventful and transpire in the blink of an eye. But in the laboratory these transitions afford a unique view of how experience is transformed from the perceptually grounded consciousness of wakefulness to the hallucinatory simulations of dreaming. The present review considers imagery in the sleep-onset transition"microdreams" in particular —as an alternative object of study to dreaming as traditionally studied in the sleep lab. A focus on microdream phenomenology has thus far proven fruitful in preliminary efforts to (i) develop a classification for dreaming's core phenomenology (the "oneiragogic spectrum"), (ii) establish a structure for assessing dreaming's multiple memory inputs ("multi-temporal memory sources"), (iii) further Silberer's project for classifying sleep-onset images in relation to waking cognition by revealing two new imagery types ("autosensory imagery," "exosensory imagery"), and (iv) embed a potential understanding of microdreaming processes in a larger explanatory framework ("multisensory integration approach"). Such efforts may help resolve outstanding questions about dream neurophysiology and dreaming's role in memory consolidation during sleep but may also advance discovery in the neuroscience of consciousness more broadly.
\end{abstract}

Key words: sleep and dreaming; mind wandering; hypnagogia; sleep onset; binding and multisensory integration; imagery

\section{Limits of Traditional REM Sleep Dream Studies}

The discovery that vivid dreaming accompanies recurrent bouts of rapid eye movement (REM) sleep (Aserinsky and Kleitman, 1953; Dement and Kleitman, 1957) was heralded as a major methodological breakthrough for the study of dream neurophysiology. The advantages were clear: investigators now possessed a method that gave immediate and predictable access to the vast reservoir of oneiric activity; activity that could be recorded in near real time with little distortion and under controlled conditions. Researchers were quick to exploit REM sleep to address fundamental questions about dreaming such as: "Is there a biological need for dreaming?" and "How does dreaming vary with sleep stage?" And although some progress was made using these laboratory methods, many fundamental questions remain unanswered. In particular, questions about the neurophysiological correlates of dreaming were not satisfactorily resolved, despite repeated attempts to assess both macro- and microphysiological sleep events. Evidence supporting psychophysiological isomorphism was so scant that Foulkes (1985, p. 4), a leader in the area, abandoned psychophysiological studies as moribund in favor of a more strictly cognitive framework.

Some insights were gleaned from neuropsychological assessments of brain-lesioned individuals (Solms, 1997), but the relationships uncovered remain imprecise and fall short of clarifying either the brain networks that sustain dreaming or the measures that reliably index it. Many studies did attempt to link dream recall or dream content attributes with specific neurophysiological indicators, most commonly with electroencephalograpy (EEG) measures, but such studies provide mixed results at best. For example, EEG correlates of remembering dreaming after REM sleep awakenings vary widely, from power reductions in alpha (Bertolo et al., 2003; Esposito et al., 2004; Chellappa and Cajochen, 2013), delta (Esposito et al., 2004), frontal beta (Chellappa et al., 2011), and 4- to 14-Hz activity (Lehmann et al., 1981), to power increases

Received: 12 August 2016; Revised: 21 November 2016. Accepted: 21 December 2016

(C) The Author 2017. Published by Oxford University Press.

This is an Open Access article distributed under the terms of the Creative Commons Attribution Non-Commercial License (http://creativecommons.org/ licenses/by-nc/4.0/), which permits non-commercial re-use, distribution, and reproduction in any medium, provided the original work is properly cited. For commercial re-use, please contact journals.permissions@oup.com 
in 40-Hz (Llinas and Ribary, 1993), occipital beta (Chellappa et al., 2011), and theta (Marzano et al., 2011; Scarpelli et al., 2015). Studies attempting to uncover EEG signatures for specific dream qualities were quite uncommon (e.g. Nielsen and Chénier, 1999).

This discovery shortfall in dream neuroscience may have been due, paradoxically, to an overabundance of phenomenological and neurophysiological information. Researchers attempted to quantify, in parallel, extended multisensory sequences of dreaming on the one hand and multifactorial arrays of neurophysiological signals on the other. Yet, approaches that attempt to align such complex phenomena are largely unstandardized, with little agreement on what levels of description and types of measurement are appropriate to assess them. This is, ultimately, an unresolved problem of isomorphic mapping (Nielsen, 2000) or, more recently, of delineating the neural correlates of consciousness (Hohwy and Bayne, 2015).

The pitfalls of such a multicomponent undertaking are too numerous to detail here but they include factors such as reliance on subjects untrained in the introspective reporting of dreams, failure to control for inaccuracies in recalling and reporting long dream sequences, assessment of EEG signals in the frequency (not the time) domain, use of broad-band EEG definitions and use of a limited number of EEG leads, among others.

In this regard, some recent studies have shown progress by focusing on increasingly brief dream events and more precise neurophysiological signals (see review in Nir and Tononi, 2010). Notably, machine learning models have been applied to visual cortical functional magnetic resonance imaging (fMRI) activity to extract patterns that accurately predict visual elements in sleeponset dreams (Horikawa et al., 2013). Other promising approaches (Andrillon et al., 2015) demonstrate similarities in the intracranial, single-neuron activity of rapid eye movements and waking percept-driven eye movements. One approach that uses multiple, serial awakenings (Germain and Nielsen, 2001; Noreika et al., 2009, 2015; Stenstrom et al., 2012; Siclari et al., 2013; Nieminen et al., 2016) has shown particular promise for expediting the collection and assessment of sleep-onset imagery and could complement the microdreaming method espoused here. Serial awakening involves the collection of multiple reports following awakenings contingent upon either experimenter-determined "neuro-centric" criteria, e.g. following randomly presented tone stimuli (Siclari et al., 2013) or target EEG configurations (Foulkes and Vogel, 1965; Germain and Nielsen, 2001; Stenstrom et al., 2012), or "phenomeno-centric" criteria, i.e. subjects themselves signaling occurrences of imagery (Germain and Nielsen, 2001; Noreika et al., 2015). Nieminen et al. (2016) combined serial awakenings with transcranial magnetic stimulation (TMS) to show that TMS during non-REM (NREM) sleep evokes smaller negative deflections when there is recall of dreaming than when there is no recall; in fact, deflection amplitude correlates negatively with dream report length. These studies have brought several new neural activity measures into play, e.g. phase-locked oscillations (Nieminen et al., 2016) and dimension-of-activation (Noreika et al., 2015) but the elaboration of phenomenological features continues to lag behind. It is precisely here that the microdreaming approach, with its emphasis on detailed phenomenological description, may contribute most to the ongoing development of second-person approaches.

In sum, early laboratory methods of REM-period awakenings for sampling dreaming have not resulted in a substantially clearer portrait of dreaming's neurophysiological underpinnings. The overabundance of information and an intractable isomorphic mapping problem failed to demonstrate reliable relationships between these two domains of measurement.
Newer methodologies targeting more discrete events show promise in resolving this problem-although phenomenological advances are disproportionately needed.

\section{An Alternative Approach: Microdynamic Neurophenomenology}

Accordingly, discovery in dream science may benefit from neurophenomenological approaches such as those applied to uncovering the neural correlates of consciousness (Thompson and Varela, 2001; Lutz et al., 2002; Garrison et al., 2013). For example, one such approach (Petitmengin and Lachaux, 2013) employs a convergence of phenomenological and neural descriptions that are both aimed at brief cognitive events. The approach has been applied successfully to the study of waking-state visual imagery (Hamame et al., 2012) among other phenomena. The feasibility of applying microdynamic neural approaches to studies of dreaming is supported by advances in brain imaging, such as the delineation of EEG-defined microstates (Milz et al., 2015) and the development of real-time fMRI (Garrison et al., 2013) and highdensity EEG (Mouthon and Huber, 2015) procedures, together with increasingly precise descriptions of sleep-onset EEG events.

A few studies support the feasibility of applying microdynamic approaches to dream neurophenomenology by simplifying neurophysiological measures and targeting specific features of dream content. In one early study (Gardner et al., 1975), subjects reported, in reverse order, only the last $15 \mathrm{~s}$ of dream content, and quantified their upper and lower dream limb movements; electromyography (EMG) tracings revealed correspondences between these and real limb activities that occurred within $5 \mathrm{~s}$ of waking up. At an even finer scale of measurement (Herman et al., 1984), subjects were trained to report only the last "few seconds" of dreamed content paying particular attention to reorientations of the eyes and head, while real pre-awakening eye movements were recorded. Again, correspondences between the directionality of dreamed and real eye movements were found.

Despite these preliminary successes, the approaches remain confounded by the very richness of the dream imagery upon which their analyses are based. First, it is problematic isolating experiential units from a large array of dream imagery and fixing these to a time scale that is reliably synchronized with neurophysiological signals. A second, related, problem is the "forward momentum" of vivid dreaming: experiential events activated at one time may remain active for an indeterminate duration and continue to shape and color events arising later. This is the phenomenological equivalent of how "upstream" neural correlates of a conscious state complicate identification of the current neural correlates of that state (Hohwy and Bayne, 2015). Third, the multisensory, multicomponent nature of rich dream imagery raises the difficulty of identifying discrete channels of information to match with neural events; different channels (e.g. visual, auditory, kinesthetic, etc.) are typically intertwined and may be wholly or partially associated with a given neural measure (e.g. EEG power). These confounds are only amplified as dream imagery becomes more vivid, extended, emotional, and multisensory-as is common for late night REM dreams. In contrast, images occurring at the very brink of sleep onset are easier to pinpoint temporally, are less influenced by forward momentum, and are simpler in structure-all features that facilitate identifying their neural correlates.

We demonstrated the feasibility of assessing microdreams in a laboratory protocol (Germain and Nielsen, 2001) in which subjects signaled precisely when brief sleep-onset images appeared in awareness. Specific relationships between image 
sensory modality, and EEG frequency and topography $9 \mathrm{~s}$ prior to the images were found; kinesthetic images possessed higher delta power in frontal regions (Fp1, Fp2, F3, Fz, F8) than did visual images, which were linked to higher delta power in mainly left central and temporal leads (F7, C3, Cz, T3, T5). Phenomenological precision in this study was relatively crude (subjective ratings of sensory imagery), but feasibility of the method was nonetheless validated. Similarly, this phenomenocentric approach was validated in a study of a single highly trained subject who reported multiple-sleep-onset images (Noreika et al., 2015); purely linguistic images were accompanied by higher left alpha and gamma power and other perceptual images by higher right beta power.

In sum, rather than grappling with the overly large data sets of traditional research, dream science could benefit from focusing a microdynamic lens on the neurophysiological fluctuations that accompany the much briefer images of sleep onset. We developed a first-person self-observational approach to study sleep-onset imagery (Nielsen, 1995) and subsequently adapted it to a secondperson approach for use with untrained laboratory subjects (Germain and Nielsen, 2001). This first-person method is used in the present work to highlight the phenomenological advantages of a focus on microdream imagery. Following a brief review of the spectrum of sleep-onset imagery, I demonstrate how microdream images in particular can be exploited to more efficiently identify the defining attributes of hallucinatory content, to uncover multiple memory sources, including previously unrecognized sensory sources, and to model imagery formation processes.

\section{The Spectrum of Sleep-Onset Imagery}

Multiple studies (see reviews in Oswald, 1962; Schacter, 1976; Mavromatis and Richardson, 1984; Vogel, 1991) attest to the presence of vivid dreams during the sleep-onset transition (Ogilvie, 2001); dreams that vary considerably in structure, specificity, length, clarity, and presence or absence of different sensory modalities (Fig. 1). Both anecdotal and empirical studies identify a spectrum of imagery ranging from elaborate and dreamlike to simple and near-subliminal. This spectrum includes images that appear to arise squarely out of the waking state. At one extreme are the complex elaborate dreams that accompany sleep-onset REM (SOREM) and partial SOREM episodes. SOREM episodes are far from rare; $13-23 \%$ of normal subjects exhibit at least one episode on the Multiple Sleep Latency Test (Bishop et al., 1996; Singh et al., 2006). Partial SOREM episodes, during which some (but not all) signs of REM sleep are visible, are also common; episodes of muscle atonia-a defining feature of REM sleep-are particularly likely to occur at sleep onset, a phenomenon referred to as "muscle atonia in NREM sleep" (Werth et al., 2002; Tinguely et al., 2006). Less complex and elaborate dream imagery occurs in NREM Stage 1 (N1) and Stage 2 (N2) sleep. Recall of any item of dream imagery is high at these times, 90-98\%, for sleep-onset stages N1 and N2 (Foulkes and Vogel, 1965; Vogel, 1991); even higher than the average level (80\%) for nocturnal REM sleep (Nielsen, 2000). However, more complex dreaming, defined as involving hallucinated dramatic episodes, is more scarce from N1 and N2 than from REM sleep: $31-76 \%$ (Vogel, 1991). Nonetheless, there are very few qualitative differences between N1/N2 and REM mentation (see review in Vogel, 1991). At the other extreme of this spectrum are the microdream images most suitable to a microdynamic analytic approach. These tend to occur during drowsiness and waking reverie and, although their brevity renders them less complex, less affectively charged, and less socially and narratively developed than images from elsewhere on the spectrum, they nonetheless share the core pseudohallucinatory qualities of such imagery and are particularly well suited for study of these qualities.

More detailed analysis of the physiological changes accompanying sleep-onset imagery (Tanaka et al., 1998a, 1998b; Hayashi et al., 1999) led to a subdivision of standard wake (W), N1, and N2 stages into a progression of nine, more precise, EEG-based substages (Fig. 2, left panel). The likelihood of recalling dream mentation is present to some degree even at sub-stage 1 and increases progressively up to sub-stage 5 (Fig. 2, upper right). Assessment of sensory attributes (Fig. 2, lower right) reveals that visual quality is high for all sub-stages, but plateaus at sub-stage 5 when all other sensory attributes diminish. Together, the results suggest that dreamlike imagery is particularly apt to arise early in sleep onset when alpha drops out and theta appears (sub-stages 4/5). Our own study (Germain and Nielsen, 2001), which used a phenomeno- rather than a neuro-centric method for sampling spontaneously arising images, confirmed this: the vast majority of signaled images ( $93.4 \%$ or $112 / 120$ ) arose from sub-stages $4 / 5$.

Finally, dreamlike imagery is sometimes reported when objective and subjective measures indicate wakefulness; almost $25 \%$ of "waking" imagery reports in two studies were qualitatively similar to REM dreams-e.g. bearing hallucinatory imagery (19\%), regressive content $(25 \%)$, or a total loss of contact with external reality (15\%)-and were distinct from waking mentation such as mindwandering or being lost in thought (Foulkes and Scott, 1973; Foulkes and Fleisher, 1975). As these studies remain unreplicated, it is unknown whether the observed images are distinct attributes of waking state imagery or, in fact, microdreams for which the sleep signs were too subtle to quantify with methods available at the time. They may have occurred during "microsleeps," which are sleep intrusions as brief as a fraction of a second (Tirunahari et al., 2003). Microsleeps are associated with localized deactivations of the brain in some regions (thalamus, posterior cingulate, occipital cortex) and localized activations in others (frontal, posterior parietal, parahippocampal), and display the smallest brain changes when they are the briefest in duration (0.5-5 s) (Poudel et al., 2014). Subjects commonly-sometimes adamantly-deny having slept during microsleep episodes despite clear electrophysiological (e.g. EEG) and behavioral (e.g. response failure) evidence to the contrary (Oswald, 1962, pp. 63-65).

\section{Conclusion: an oneiragogic spectrum}

Windt (2010, 2015), better than any previous author, delineates a comprehensive framework for describing the variety of sleep-onset imagery, what she dubs the spectrum of "oneiragogic experience." This new term refers to the progression of phenomenological events leading from drowsy wakeful perception to full-fledged dreaming (Table 1, items 1-5) or "the trajectory underlying the shift in self-location from a perceptual to a hallucinatory (or dreamlike) reference frame" (Windt, 2015, p. 536). In other words, one's sense of immersion in space and time ("here" and "now") shifts progressively from a veridical framework (the real world) to a hallucinatory framework (the dream world). Windt considers the oneiragogic spectrum analogous to, but distinct from, the hypnagogic state, which describes a progression of physiological changes from wake to sleep. The oneiragogic spectrum forms part of a broader conceptual framework for dream phenomenology, the immersive spatiotemporal hallucination (ISTH) model (see Windt, 2015, chapter 11). Five dimensions are proposed to constitute the oneiragogic spectrum; I suggest adding to these a sixth dimension, kinesis, to describe the experience's movement quality (Table 1, item 6).

Whereas the concept of microdreams has been used to describe all sleep-onset imagery (see review in Oswald, 1962), its 
Spectrum of oneiragogic experience in the sleep onset transition: imagery types and associated sleep/wake stages

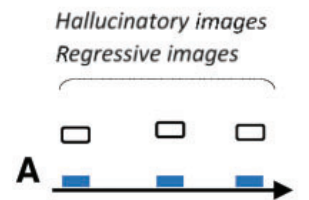

Waking reverie

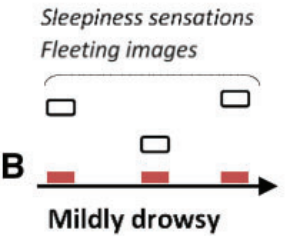

Mildly drowsy

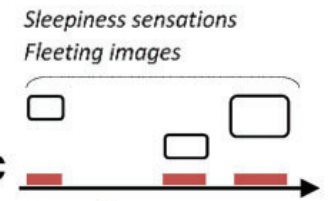

Very drowsy

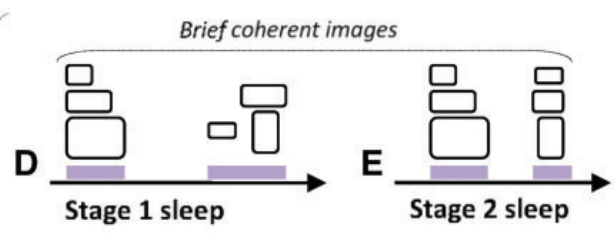

$\mathbf{F}$

Partial SOREM sleep

Brief coherent images

Longer images

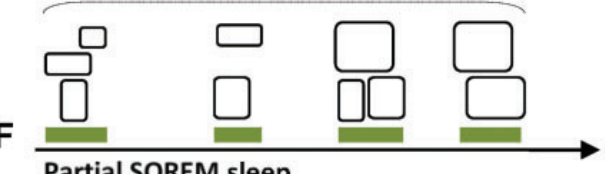

Full-blown dream imagery

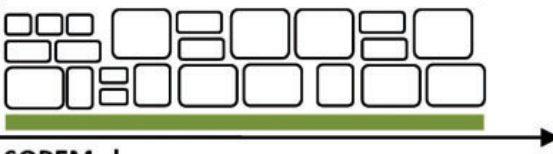

SOREM sleep

\section{$\underbrace{}_{\sim 1 \mathrm{sec}}$}

Sleep Onset Transition

Figure 1. Varieties of oneiragogic experience during the sleep-onset transition. Experiences of increasing complexity occur during passage through different sleep-onset states as described in the text. The least complex events are presumed to arise in microsleeps that occur during (A) waking reverie (blue bars) or (B) mildly or (C) very drowsy wakefulness (red bars) and more complex events during (D) Stage 1 and (E) Stage 2 NREM sleep (purple bars). Longer images and full-fledged dreaming occur during either (F) Partial SOREM sleep, when only some signs of REM sleep (e.g. muscle atonia) are visible (green bars), or (G) SOREM sleep, when all REM sleep signs are visible.

\begin{tabular}{ccc}
\hline $\begin{array}{l}\text { R\&K } \\
\text { stage }\end{array}$ & $\begin{array}{c}\text { Hori } \\
\text { stage }\end{array}$ & EEG composition \\
\hline $\mathrm{W}$ & 1 & Alpha wave train \\
$\mathrm{W}$ & 2 & Alpha wave intermittent $(>50 \%)$ \\
$\mathrm{S} 1$ & 3 & Alpha wave intermittent $(<50 \%)$ \\
$\mathrm{S} 1$ & 4 & EEG flattening \\
S1 & 5 & Vertex sharp wave solitary \\
S1 & 6 & Vertex sharp wave train/burst \\
S1 & 7 & Ripples of low-voltage theta waves \\
S1 & 8 & Vertex sharp wave and incomplete spindle \\
S2 & 9 & At least one complete spindle \\
\hline
\end{tabular}
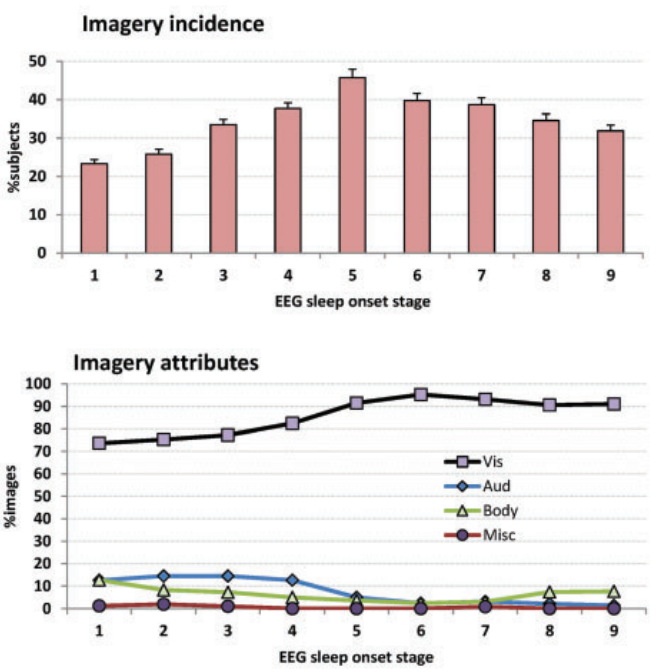

Figure 2. Left panel: Definitions of nine Hori sleep-onset sub-stages (Hori stage) in relation to standard Rechtschaffen and Kales sleep/wake stages (R\&K stage). Right panel: Percentages of subjects reporting dreams (upper panel) and sensory attributes of reported imagery for Hori sub-stages (from Hori et al., 1994).W = wake; S1 = Stage 1; S2 = Stage 2. Note that visual imagery can occur in the earliest sub-stages.

use is clearly inappropriate for more complex imagery forms, such as those reported from REM and partial REM episodes. I use the term microdream in a more limited sense to refer to images falling on the simpler extreme of the oneiragogic spectrum. In sum, the oneiragogic spectrum improves upon the phenomenological triage of imagery types in facilitating identification of particularly brief images of interest to the microdynamic approach.

\section{Microdreaming}

Because existing studies have typically treated sleep-onset imagery as of a single type, only rarely are simpler images contrasted with more complex ones, despite the fact that differences in dream report length have remained a central methodological concern of researchers who compare REM and NREM dream reports (Hunt 
Table 1. Summary of Windt's oneiragogic experiential spectrum including five constitutive immersive spatiotemporal hallucination (ISTH) dimensions and a proposed amendment

\begin{tabular}{|c|c|c|c|}
\hline ISTH dimension & Description & $\begin{array}{l}\text { Simple extreme } \\
\text { (closer to waking) }\end{array}$ & $\begin{array}{l}\text { Complex extreme } \\
\text { (closer to dreaming) }\end{array}$ \\
\hline 1. Visuospatial scene & $\begin{array}{l}\text { A sense of being located at a specific point in } \\
\text { a larger spatial expanse ("here") }\end{array}$ & Isolated, static & $\begin{array}{l}\text { Dynamic, prolonged, } \\
\text { immersive }\end{array}$ \\
\hline 2. Phenomenal embodiment & $\begin{array}{l}\text { A sense of bodily awareness that progresses } \\
\text { from passive observation to active } \\
\text { participation }\end{array}$ & $\begin{array}{l}\text { Partial body aware- } \\
\text { ness (e.g. sleepi- } \\
\text { ness feelings) }\end{array}$ & Full-body awareness \\
\hline 3. Temporal reference frame & $\begin{array}{l}\text { A sense of being located in a specific moment } \\
\text { within a succession of moments ("now") }\end{array}$ & $\begin{array}{l}\text { Brief, isolated } \\
\text { instant }\end{array}$ & $\begin{array}{l}\text { Prolonged, organized } \\
\text { narrative }\end{array}$ \\
\hline 4. Waking memory sources & $\begin{array}{l}\text { Integration of recent with increasingly re- } \\
\text { mote memory sources }\end{array}$ & $\begin{array}{l}\text { Recent, episodic } \\
\text { memories }\end{array}$ & $\begin{array}{l}\text { Remote, abstract, } \\
\text { but semantically } \\
\text { related memories }\end{array}$ \\
\hline 5. Autobiographical historicity & $\begin{array}{l}\text { A sense that an image is part of one's auto- } \\
\text { biographical experience/memory } \\
\text { ("recallability") }\end{array}$ & $\begin{array}{l}\text { Barely graspable as } \\
\text { own experience, } \\
\text { difficult to recall }\end{array}$ & $\begin{array}{l}\text { Integration in auto- } \\
\text { biographic mem- } \\
\text { ory, easily recalled }\end{array}$ \\
\hline $\begin{array}{l}\text { 6. Spatiotemporal kinesis } \\
\text { (proposed) }\end{array}$ & A sense of dynamism, movement & $\begin{array}{l}\text { Ultra-brief, incipient, } \\
\text { or isometric } \\
\text { movement }\end{array}$ & $\begin{array}{l}\text { Complex } \\
\text { movements }\end{array}$ \\
\hline
\end{tabular}

et al., 1993). The only systematic studies that attempt to identify the simplest forms of images are self-observational in nature. From a set of over 250 sleep-onset images recorded under either laboratory or naturalistic conditions, and for which a preliminary phenomenological description is presented elsewhere (Nielsen, 1992, 1995), a subgroup of images referred to as "fleeting images" was identified that I here consider a type of microdream. Some examples appear in a later section. I distinguished these brief images from "fully-formed" images in respect to their timing, duration, simplicity, ineffability, and association with sleepiness feelings. In short, fleeting images were previously found to:

- occur in the very earliest stages of a self-observation trial, i.e. during mild drowsiness;

- be so brief that they often require an established intent (if not introspective training) to observe;

- possess primarily unimodal sensory content;

- frequently fall outside of the normal range of experience, thus defying easy description;

- be accompanied by subtle feelings of falling asleep.

Fully formed images, in contrast, were found to:

- occur later in the sleep-onset transition;

- be longer and easier to recall;

- possess a more complex, multimodal structure;

- be easier to describe;

- often mask accompanying sleepiness feelings.

Using the oneiragogic spectrum terminology, fleeting images may now be described more specifically to:

- implicate an isolated spatiotemporal location ("here");

- include a sense of body awareness, however, ephemeral (e.g. sleepiness feelings);

- occur in a discrete instant ("now");

- draw upon a few recent, episodic memories (e.g. ongoing sensory stimuli);

- often be barely graspable and difficult to recall;

- possess a subtle kinetic quality, i.e. sensed movement of self or setting.

Fully formed images, in contrast, more closely resemble REM dreams: they are spatiotemporally elaborate (multimodal), are more embodied and temporally extended, draw upon a wider swath of remote memory elements, are more fully integrated into one's historical context (easily recalled), and are more obviously dynamic in character.

There is still no validated method for distinguishing fleeting microdreams from fully formed dream images, but the oneiragogic spectrum - possibly with the addition of the kinesis dimension-provides a thorough framework for drawing such distinctions clearly and reliably. With further development, it could serve as a standardized tool for classifying the phenomenological attributes of sleep-onset imagery. While extensive training in self-observation is not essential for collecting microdreams, accuracy can be improved by following an "upright napping" procedure (Nielsen, 1992, 1995). In two studies using upright napping, a maximum of 7 and $13 \mathrm{im}$ ages/session, respectively, were recorded. In general, sitting upright prevents one from slipping into deep sleep thereby facilitating accurate recall. Immediate review and recording of imagery context, phenomenological details and memory sources is critical. Establishing an observational intent and applying structured rating scales to images is also valuable (Nielsen, 1995). Note that practice may well increase the likelihood of observing images, just as practice improves recall of morning dreams (Aspy, 2016), but it remains to be studied whether practice changes microdream content qualitatively.

\section{Example microdream}

An example microdream recorded in our sleep laboratory is shown in Fig. 3. The timing of this primarily kinesthetic image was signaled while falling asleep with a hand-held switch. The signal immediately follows the image, allowing its associated neurophysiological changes to be examined. Changes include occipital alpha decrease, REM atonia-like EMG decrease (Werth et al., 2002) and, possibly, a brief rapid eye movement.

The image was brief and unusual in that it consisted of apparent sensations of touch ("knocking") and motion ("moving") that seemed to occur inside the head. On the oneiragogic spectrum, it displays: (i) rudimentary spatiality ("inside"), including vague visuospatiality ("someone else's head"), (ii) minimal embodiment ("head"), and (iii) minimal temporal extension ("[the knocking] is moving"). There was no report of (iv) memory 


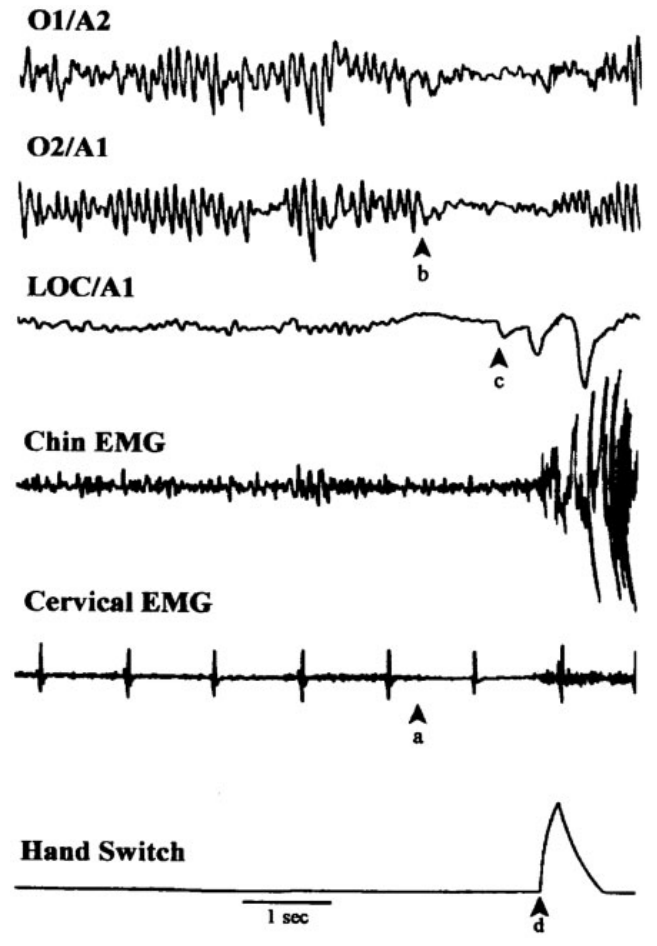

Figure 3. Sample microdream recorded using a phenomeno-centric signaling procedure. The image ("I feel a knocking inside my head and a sense that it is moving; also a vague visual impression of seeing someone else's head") was primarily kinesthetic and kinetic with a weak visual component. The physiological correlates define a microsleep about $1 \mathrm{~s}$ in duration, including: (a) an abrupt decrease in cervical and chin EMG, (b) a sudden bilateral decrease in occipital alpha (Hori sub-stage 2) and the appearance of a low amplitude, slow eye movement on the LOC/A1 channel. This pattern changes abruptly to wakefulness (alpha with movement) at (d) when the end of the image is signaled. The appearance of (c) two brief rapid eye movements just prior to the (d) signal may reflect an oculomotor correlate of the image or simply reflect an awakening reaction. Cardiac activity is a steady $60 \mathrm{bpm}$ (from Nielsen, 1995).

sources, but (v) minimal historicity is suggested by the difficulty in recall ("vague visual impression"). Finally, the image possesses (vi) a clear spatially kinetic character in that the sensations changed dynamically over time ("knocking," "moving"). Note that although the image memory sources were not reported or probed, the ready accessibility of these sources was previously demonstrated (Nielsen, 1995). Memory sources are considered in a later section.

Such simple oneiragogic experiences can be mined for evidence of features that define dreaming's phenomenal core. These features are likely to be more easily discernible in simple images because these lack the common, albeit nonessential, features so typical of complex REM sleep imagery, e.g. emotion, visual/auditory details, or narrative structure. Windt (2015, pp. 517-520) argues that dreaming, including especially sleep-onset dreaming, may be purely spatial in nature, an argument bolstered by the observation that congenitally blind subjects report vivid dreaming despite their total absence of visual imagery in wakefulness (Kerr et al., 1982; Kerr and Domhoff, 2004). The next section argues that spatiotemporal kinesis may also be a defining feature of microdreaming.

\section{Spatiotemporal kinesis}

The kinetic attributes of a living organism are its capacity to move or react in its environment, usually in response to sources of stimulation. Analogously, an image's kinetic qualities refer to its inherent dynamism, vitality, or moment-to-moment changeability within its hallucinatory context-and possibly also in response to its various memory sources of stimulation. Full-blown dreaming is clearly kinetic in this respect, rich with immersive self-movement, character actions and environmental flux that can all be linked to memory source elements. Microdreams, too, express this feature to some degree-even in their simplest, most ephemeral forms-and in this respect their phenomenal core is one with that of dreaming. Kinesis manifests most often as a realistic apparent movement of the self (or part of the self) or of another character, or of an apparent movement felt to take place inside the body. It also manifests as apparent movement of the environment in proximity to the self. But it may even occur in ways that are less obviously related to visuospatial modulation. For example, images containing vivid isometric tension or sensations of an imminent action or impending contact with some object or person may reflect a minimal form of spatial kinesis. In this respect, the present description of microdreams differs somewhat from that of Windt, who considers the simple extreme of microdreaming to be visuospatial stasis. Rather, kinesis may be as integral to the immersive nature of microdreams as it is to completely hallucinatory dream experiences. Whether all microdreams are kinetic, and how to quantify kinesis in its simplest forms are, therefore, fundamental empirical questions requiring further study.

\section{Microdream memory sources}

The study of microdreams offers unique possibilities for developing experimental methods to clarify how and why images are formed. These possibilities hinge on identifying an image's memory associations, which are widely assumed to index the memory sources from which an image derives (Freud, 1900; Nielsen and Stenstrom, 2005). The following sections deal with three advantages of memory source analysis that microdreams confer, including how such work may contribute to the development of experimental methods for investigating image formation processes.

\section{Access to multi-temporal memory associations}

The memory sources of microdreams are few but readily accessible. Because microdream images are brief and occur in such close proximity to wakefulness, attention can be directed quickly and flexibly to identifying their source memories. Using a self-observational approach, we previously demonstrated both the feasibility of identifying memory sources and that these sources are relatively few in number (Stenstrom et al., 2012). Figure 4 shows all memories associated with 31 sleep-onset images collected from a single subject, classified by the memories' times since occurrence. The number of memories for each image is clearly restrained: on average $2.6 \pm 1.1$ (range 1-5) per image (Contrast this with Freud's memory source analysis of his "Irma's Injection" dream: 332 words of dream and 4130 words of memory sources). For a majority of these images, memories were drawn simultaneously from both recent and remote time periods, a finding consistent with other research for dream imagery (Verdone, 1965). Further, systematic variations in the temporal distance of these memories from the target image suggests that their access may have been modulated by time of night and, possibly, day of week factors. For example, on 


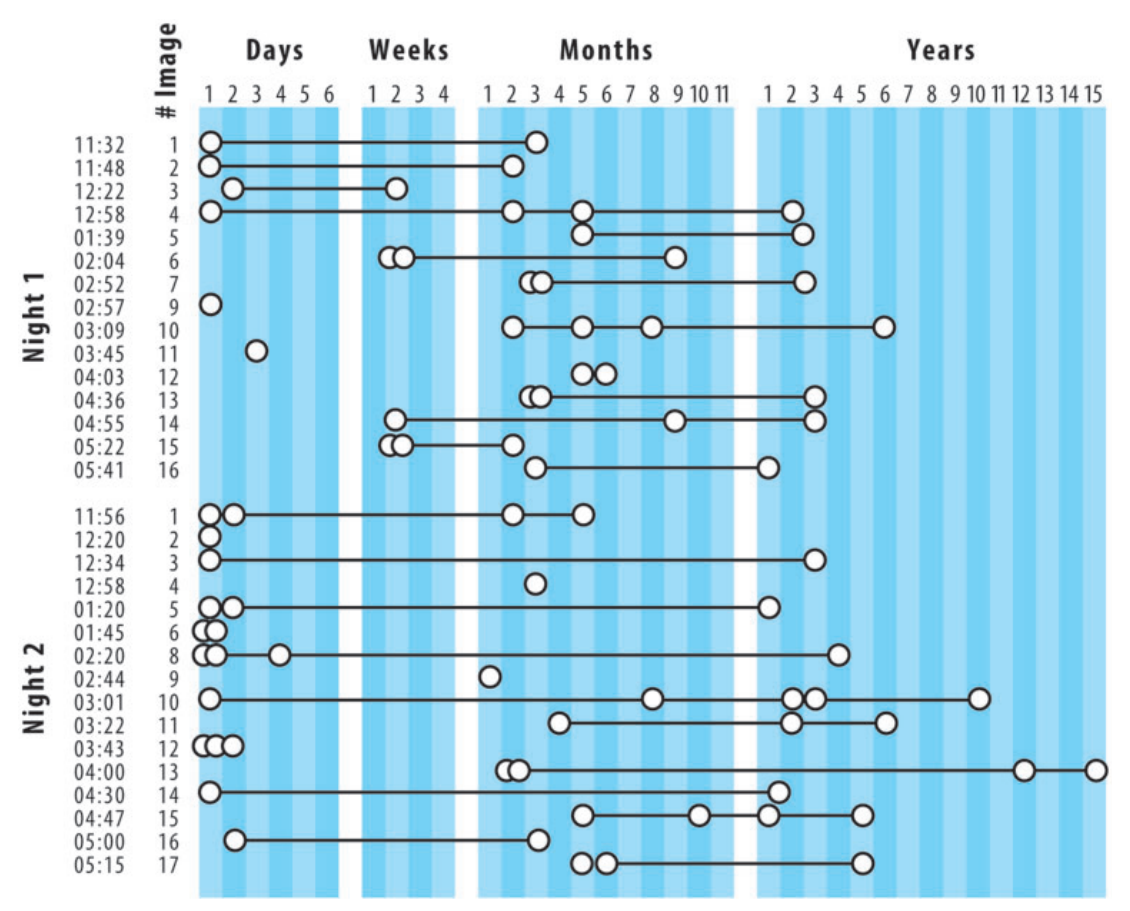

Figure 4. Temporal distances between sleep-onset images and their memory sources collected with a serial awakening method over two nights from a single subject. Circles represent memory sources; black lines indicate that sources are for the same image (from Stenstrom et al., 2012).

both Nights 1 and 2 memories for elements that were encountered just prior to sleep (e.g. lab technician, EEG equipment) were clearly reported for the first four images of the night; yet, for Night 1 these very recent memories tended to diminish for later night images, while for Night 2 they persisted. Such variation may mean that circadian factors modulate access to memory sources (for review, see Nielsen, 2011) or that habituation to the laboratory and the self-observational method takes place before access to memory sources is stable. In either event, this case study illustrates the ease of both collecting memory sources at sleep onset and evaluating their temporal distances from a target image.

Sample memory source analysis. The following example describes a microdream's memory sources arising from multiple time periods and illustrates a hypothetical model that could explain formation of the image (for analysis, see Nielsen, 1995). The proposed multi-temporal classification of memory sources consists of four categories differentiated by their temporal distance from the target image:

1. Immediate memory: thoughts, perceptions, and sensations that are ongoing or occurred only moments before image onset. Might include sensations of pressure in the arms, legs, or neck that manifest as arm, leg, or head imagery.

2. Short-term memory: experiences that occurred minutes to hours prior to image onset. Relatively easy to identify, occurring only a short time before the observation session.

3. Medium-term memory: experiences that occurred one or more days prior to the image. Include memories from the previous day (day-residue effect; Freud, 1900; Nielsen and Powell, 1992) and from 6 to 8 days earlier (dream-lag effect; Nielsen et al., 2004; van Rijn et al., 2015). These sources may be difficult to retrieve but access can be facilitated by a systematic review of salient events from the previous week.
4. Long-term memory: experiences that occurred weeks, months, or years prior to the image. May be difficult to identify and have been investigated only minimally. Are often rooted in declarative memory and resemble "semantic knowledge" memory sources (Cicogna et al., 1991).

Figure 5 illustrates a 1-s microdream collected by the author in a naturalistic setting (seated with left elbow resting on table, knuckles supporting chin), immediately recorded in detail (right panel) and depicted with components listed in chronological order (left panel, $\mathrm{X}$-axis, bottom row). Its associated memory elements were also recorded and classified according to the fourpart temporal distance scheme (Y-axis). These sources are arranged in a hypothetical chronological sequence with causal influences indicated by arrows.

A qualitative comparison of the image elements and memory sources suggested that the image is a close, but not exact, reproduction of a distinctive episodic memory fragment, i.e. performing a quasi-reflexive arm movement during a volleyball game 6 days earlier. This fragment possesses a sequential structure that is mirrored in the microdream: the visual approach of the ball, the rapid motor response of the arm, and the cutaneous feedback from contact with the ball, which were all experienced a single time during a real volleyball exchange, are represented in slightly altered form but in the same chronological sequence. This primary memory fragment is modified by several secondary fragments such that the final image does not faithfully depict the episodic memory, e.g. the original arm movement became completely reflexive in the image; the ball's trajectory was altered; the ball's size and weight became appropriate to a larger, lighter beach ball, etc. Such transformations of episodic elements may reflect the piecemeal nature of episodic memory storage (e.g. Brady et al., 2013) or the influence of consolidation mechanisms that transform episodic into semantic memories (e.g. Stickgold, 2002). 


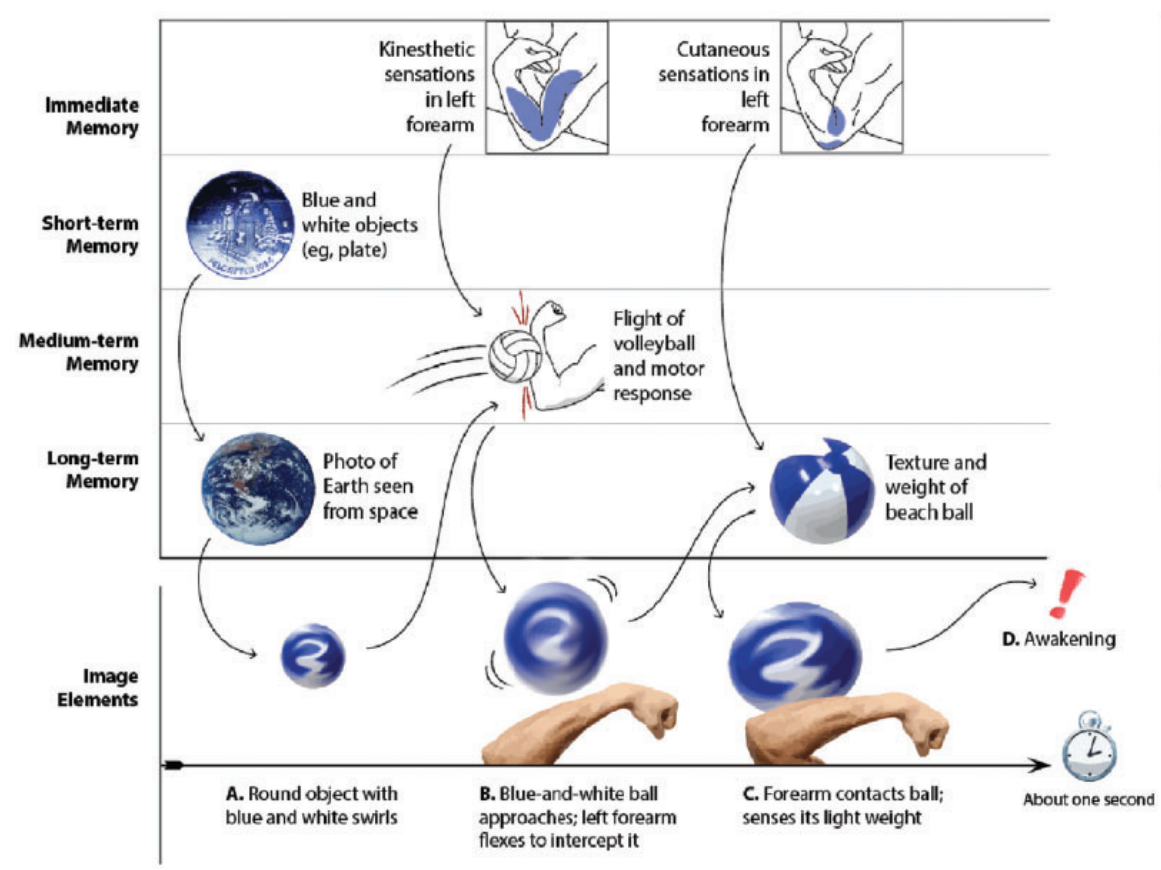

Image: / see a small blue-and-white object far off to my left. Its colors are very bright and form a swirled pottern. It suddenly and unexpectedly flies towards me, horizontally but with a slight arc. It was as if someone had thrown it at me. Close to me it is about the size of a basketball. I make a quick, reflexive movement with my left arm as if to strike or intercept it. For an instant I feel a sensation on the upper part of the elbow and forearm as the ball makes contact with me. From this I perceive that the ball is much lighter and thinner in consistency than I had expectedlike a beach ball. Though I expect it to bounce, it stays in my arms as if I had caught it there. However, I wake up abruptly at the moment of contact, before anything else can happen. I was surprised by the lmage.

Figure 5. Multi-temporal description of memory sources associated to a single, 1-s microdream (right panel) and hypothetical model of their realtime combination during image formation. Four categories of memory sources (Y-axis) combine to produce elements of the microdream sequence (X-axis). Arrows indicate possible causal influences among elements via a "transformative priming" process. The image is proposed to unfold in three steps: (A) several short-term and one long-term memory elements combine to produce a round, blue-and-white object seen at a distance; (B) the prior result combines with immediate memory impressions (kinesthetic) and one medium-term memory (flight of ball, motor response) to produce a transformed image of an approaching ball and reflex motor response; (C) the prior result combines with additional immediate memory impressions (cutaneous) and a long-term memory element (beach ball) to produce a transformed image of object contact with touch feedback. Memory sources transform subsequent elements even as they trigger ("prime") them-but all very quickly and at a preconscious level. The result is a continuous de novo integration of memory sources that reflects features from all contributing memories, but which expunges other features. The primary memory element-a reflex arm movement pictured in the center of the grid-is modified by secondary elements: color, trajectory, weight, and texture are changed slightly. Transformative priming may sustain spatiotemporal coherence of the sequence, may account for an image's novel character and may explain why larger episodic memories are typically not represented (artwork courtesy: Sabrina Nielsen).

The memory sources identified by this simple descriptive analysis lend themselves to hypothetical causal models of image formation. One type, a real-time model illustrated by the connecting arrows in Fig. 5, might stipulate that memory sources are combined successively in the order shown. The notion of "transformative priming" is suggested as a basic mechanism of such real-time combination such that each memory source both triggers (primes) and merges with (transforms) a subsequent memory or imagery element. Alternatively, an off-line model might suggest that the image sequence emerges fully formed into awareness, without the need for real-time integration, i.e. memory elements are combined in the days, hours, minutes, or even seconds prior to the image but not in real time. A similar mechanism was postulated to account for some symbolic dream elements (Freud, 1900). Yet other models might be proposed that stipulate combinations of real-time and off-line influences. Figure 5 is but a starting point, intended to demonstrate that much of the evidence needed to explore such hypotheses is accessible from a thorough description of image and memory sources. And unlike previous efforts to analyze the memory sources of lengthy dream reports (Freud, 1900; Foulkes, 1978), microdreams offer a much simpler set of phenomenological observations and require a more limited number of hypothetical constructs to explain their formation.
Access to cognitive and somatic imagery sources: autosymbolic imagery

Microdreams are particularly useful for exploring the potential influences of recent memory sources to the extent that these reflect specific episodic experiences as opposed to general semantic knowledge. This is amply demonstrated by Silberer's (1951) study of autosymbolic images, or images that automatically reflect ongoing waking states, thoughts, or feelings. He suggested three types:

1. Material images: These reflect the contents of pre-imagery thoughts, such as an abstract idea (e.g. thinking about the nature of transsubjectivity) being represented by a perceptual image (e.g. dreaming of a transparent sphere with peoples' heads in it).

2. Functional (effort) images: These reflect attributes of the preimagery thinking process, such as a quality of cognitive activity (e.g. losing one's train of thought) being reflected symbolically (e.g. image of a line of text with no ending).

3. Somatic images: These reflect the influence of sensations of any kind, such as somatic sensations (e.g. taking a deep breath) being reflected metaphorically (e.g. dream of lifting a table high in the air).

Silberer suggested-but did not empirically demonstratethat autosymbolic images could be employed to examine dream 
Table 2. Chronology of events leading to an autosensory microdream (illustrated in Fig. 6)

\begin{tabular}{|c|c|c|}
\hline Stage & Event & Comments \\
\hline Context & $\begin{array}{l}\text { Lying down on L side; L thumb, index and middle } \\
\text { fingers were next to forehead while falling asleep. }\end{array}$ & $\begin{array}{l}\text { Fingers were not put in this position intention- } \\
\text { ally; I was not aware of them there until the } \\
\text { image occurred. }\end{array}$ \\
\hline Ongoing cognitive activity & $\begin{array}{l}\text { I had been imagining using my L hand to execute a } \\
\text { difficult (for me) guitar chord (Em add G\#), unsure } \\
\text { of its order in a song. }\end{array}$ & $\begin{array}{l}\text { This chord requires a pinching action by the L } \\
\text { thumb, index and middle fingers similar to the } \\
\text { squeezing needed to remove a bottle cap. A } \\
\text { difficult chord for me. }\end{array}$ \\
\hline 1. Original image & $\begin{array}{l}\text { I was using my L thumb, index and middle fingers to twist } \\
\text { off the dark blue top of a blue plastic water bottle. I saw } \\
\text { the cap between my fingers. }\end{array}$ & $\begin{array}{l}\text { I typically perform this movement with my R } \\
\text { hand. I cannot recall having done it with my L } \\
\text { hand. }\end{array}$ \\
\hline 2. Reflex/stimulus & $\begin{array}{l}\text { While twisting the cap, I felt my actual L hand fin- } \\
\text { gers twitch briefly and with the same direction of } \\
\text { movement. I also felt a touch on my forehead. }\end{array}$ & $\begin{array}{l}\text { The image } \rightarrow \text { movement } \rightarrow \text { touch sequence oc- } \\
\text { curred very quickly. I realized almost immedi- } \\
\text { ately that the touch was self-produced. }\end{array}$ \\
\hline $\begin{array}{l}\text { 3. Autosensory image } \\
\text { (somatic) }\end{array}$ & $\begin{array}{l}\text { DZ was reaching her } R \text { arm in from my } R \text { side. It was gray } \\
\text { and had very little form. I saw and felt her hand touch } \\
\text { me at the same place my fingers were resting. }\end{array}$ & $\begin{array}{l}\text { The visual details were relatively indistinct but } \\
\text { the arm's presence was very distinct. The im- } \\
\text { age occurred as I woke up. }\end{array}$ \\
\hline 4. Wake up & $\begin{array}{l}\text { The entire image } \rightarrow \text { movement } \rightarrow \text { touch } \rightarrow \text { image } \rightarrow \\
\text { wake up sequence took less than a second. }\end{array}$ & $\begin{array}{l}\text { The sequence was recalled rapidly in reverse } \\
\text { order. }\end{array}$ \\
\hline
\end{tabular}

Imagery description appears in italics.

production mechanisms experimentally. Experimental methods have, in fact, been used to influence the dreams of REM sleep (see review in Arkin and Antrobus, 1991), but Silberer's examples did not employ such experimental controls. His post hoc observations of juxtapositions between images and memory elements do not guarantee that they are causally linked. Underlying factors that straddle sleep/wake state may produce both the memory source and the closely related image that follows it. Nonetheless, some self-observations reported below do support Silberer's causality assumption. While anecdotal, these bolster the feasibility of developing more thorough experimental approaches to investigate microdreams and to model their formation mechanisms.

Silberer's focus on immediate waking state precursors to microdream imagery suggests avenues of study that could overlap related methods for assessing consciousness dissolution during, e.g. meditation (Lehmann et al., 2001) or induced unconsciousness (Mashour, 2011). To illustrate, while there is evidence that sleep-like increases in delta power and neural network fragmentation accompany propofol anesthesia (Lewis et al., 2012; Purdon et al., 2013), such studies often address only the simplest phenomenological constituents of consciousness-e.g. its presence or absence. Microdream research, with its emphasis on phenomenological exactness, could contribute substantially to developments in this domain.

\section{Access to causal factors in image formation}

To facilitate causal analysis and modeling in microdream research, I suggest broadening Silberer's three-part classification to include instances in which memory stimuli are more obviously linked causally to images. I suggest both adding a fourth type of autosymbolic imagery-"autosensory imagery"-to the list and expanding upon Silberer's third type of imagery (somatic) to include "exosensory imagery."

Autosensory imagery. An autosensory image is shaped by a selfgenerated stimulus that occurs immediately prior to the image's appearance in awareness. The self-generated stimulus usually stems from a neuromuscular event like a muscle twitch, body jerk, or nodding of the head while falling asleep but may also include sounds like snoring, wheezing, or verbalization. Such events may even be triggered by one image only to merge with a subsequent one. Thus, a particularly kinetic microdream, such as poking an object with a dream finger, may produce an overt dream-enacting behavior (finger twitch) that stimulates a sensation of touch in a different body region (adjacent skin)—a sensation that feeds into and modifies a subsequent (autosensory) image. An autosensory image involving touch could be differentiated from others as "autosomatic" in that the image's embodiment dimension is altered. Autosomatic images are the most common type I have observed although other types are possible: "autoacoustic" images for auditory stimuli (e.g. hearing one's own voice or movements) or "autovestibular" and "autooptical" images for vestibular and visual stimuli, respectively. Because of the brevity of microdreams, a dream-enacting behavior that elicits an autosensory image may also provoke an awakening even as the new image takes hold in awareness.

An example of an autosomatic image is presented in detail (Table 2, Fig. 6) to highlight its clear causal implications in imagery formation.

The example begins with a sub-example of a Silbererian type 1 (material) autosymbolic image for which a pre-sleep reverie about forming a difficult guitar chord with fingers of the left hand is followed by an image in which the same fingers unscrew a plastic bottle cap. The microdream action is further associated with twitch-like movements of the real fingers resting on, and triggering touch sensations in, the forehead. The latter sensations, in turn, evoke the autosensory image of someone abruptly appearing and touching precisely the same location on the forehead. The enactment and subsequent stimulation trigger an awakening immediately after the autosensory image enters awareness. The precise chronological order of events allows a causal connection to be inferred.

In light of this clear phenomenological sequence, the question arises as to how a self-induced cutaneous sensation produces a contextualized visual image of someone who appears to be the source of the sensation. In other words, how does a cutaneous stimulus so immediately produce its own contextappropriate visual precursor? This puzzle may be viewed as a special case of conflict in multisensory temporal ordering and 


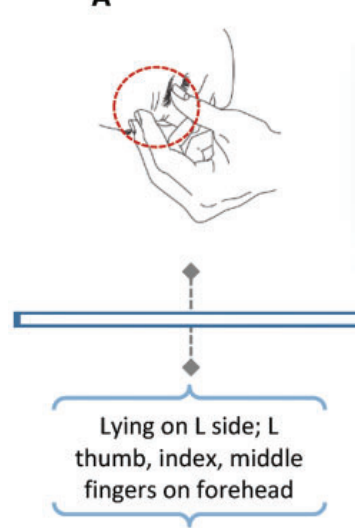

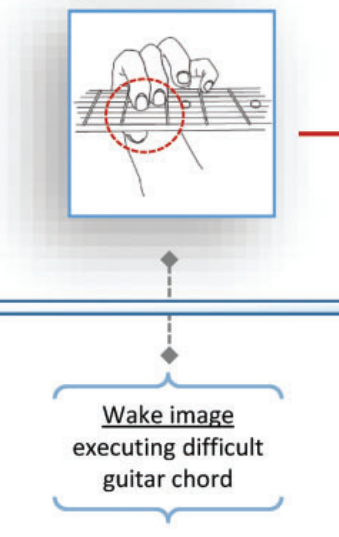

C

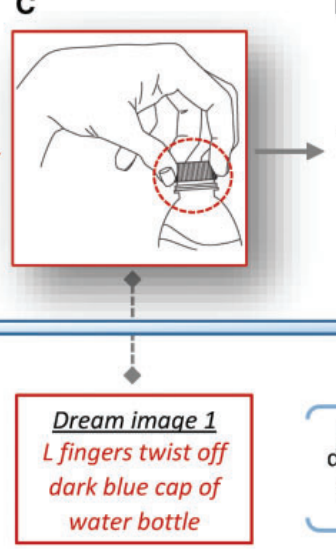

D

D

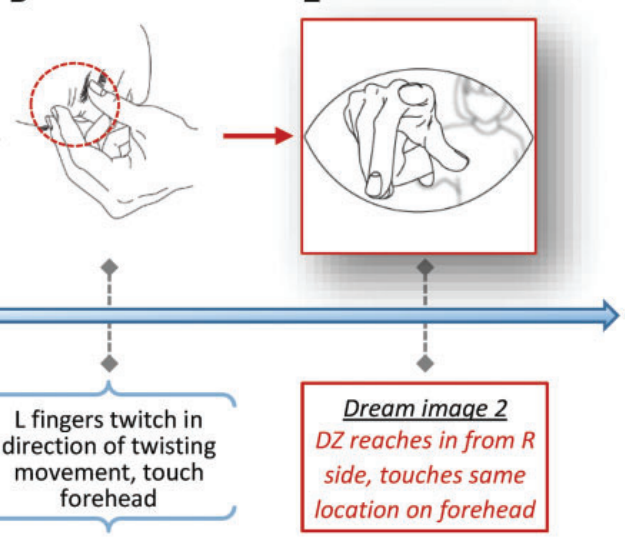

Figure 6. Chronology of events leading to an autosensory microdream. (A) The physiological context (falling asleep with fingers on forehead). (B-E) Imaginal events with verbal descriptions given below the timeline. Contextual information is in black text in horizontal parentheses; microdream imagery in red-bordered boxes and red text. See Table 2 for more details (artwork courtesy: Sabrina Nielsen).

will be addressed more fully once we have considered other instances in which external sensory stimuli causally influence microdreams, i.e. cases of exosensory imagery.

Exosensory imagery. I suggest exosensory imagery as a more inclusive category of Silberer's third type of autosymbolic imagery (somatic imagery), i.e. imagery influenced causally by external stimuli (sounds, lights, imposed body movements, cutaneous contacts, etc.). At present, examples of exosensory microdreams are anecdotal and based on fortuitous observations made under the sleep-onset conditions detailed in Table 3. External stimulations occurred unpredictably in locations as varied as an airplane in turbulence, a noisy park, and a quiet office. Before discussing the exosensory nature of these images and their implications for image formation, some of their phenomenological, ISTH-like, characteristics are described.

- Brevity (autobiographical historicity): The 18 images in Table 3 are all short-duration episodes-microdreams-despite their sometimes lengthy descriptions $\left(M_{\text {words }}=28.8 \pm 14.4\right.$; range 12-75). Most $(N=$ 14) consist of a single part, usually a single dreamed movement of a limb, character, or object, accompanied by some visual details. The other four images (\#1, \#3, \#9, \#10) consist of at most two parts: two consecutive actions or movements with visual details. In all but one of these (\#3), coherence between the two parts is discernible, but in image \#3, the second part ("cat's head emerges") seems tangential to the first part ("hand puts file in box").

- Eruptive quality (temporal reference frame; spatiotemporal kinesis): Most of the images possess an eruptive character in two separate but interrelated ways. First, they seem to erupt into awareness, eliciting surprise and a quick return to consciousness. Second, the image content hinges on an abrupt emphatic movement that seems to have been caught in mid-expression. Thus, for image \#2, the multi-colored clown is seen spinning with an energy and trajectory consistent with having just tripped violently or jumped off a trampoline. Similarly, in image \#5 a young man vaults with such velocity that he seems to have just stumbled while running, and gives the image a "caricature-like" aspect. This eruptive quality is especially true for images affected by phasic stimuli, while for some images affected by tonic stimulation (e.g. \#11-\#13) an eruptive action is less apparent than its sudden appearance in awareness. For other tonic images (\#15\#18) an eruptive action is, in fact, present.
- Instability (visuospatial scene): Many of the images depict an instability theme: their primary constituent is a vigorous, even violent, movement in a downward or circular direction. These include an arm slapping down (\#1); a clown somersaulting (\#2); a door slamming (\#4); a man tripping (\#5); a girl falling (\#7); a woman spilling a drink (\#8); a bicycle collapsing and man falling on it (\#9), a spinning ball sliding and falling (\#10); and a page falling (\#15). Image instability appears closely linked to the eruptive quality [Readers may be familiar with the common sleep-onset imagery of abruptly falling that can occur after a day of vigorous sport such as skiing (Wamsley et al., 2010). Such images both depict the self as falling and lead to an eruptive awakening, usually accompanied by an intense bodily jerk.].

- Self us. nonself focus (phenomenal embodiment): Focus of the microdreams is sometimes the movements and sensations of the self $(N=7)$ and sometimes those of other characters or objects $(N=$ 11). Accordingly, the effects of external stimuli are detected in modifications to either self (somatic) or nonself (visual, auditory) imagery attributes. In rare cases (\#17), an apparent overlap between self and nonself attributes occurs, e.g. the actions of another character are felt to be executed by the self.

Influence of external stimuli. Several important relationships between image elements and their immediately preceding external stimuli are illustrated in this collection.

Multiple sensory modalities: Images are affected by stimuli from many sensory modalities, including vestibular, auditory, visual, and various somatic stimuli (cutaneous, thermal, pain). Visual stimuli are the least prevalent. There is a fairly specific correspondence between stimulus modality and its influence on the image; in general, auditory stimuli affect sounds, vestibular stimuli affect balance, cutaneous stimuli affect touch, thermal stimuli affect perceived temperature, and so forth. Some exceptions to this general rule (e.g. \#3, \#18) point to nonspecific influences. For example, in image \#18 pain and cutaneous sensations in a limb were associated with a visual image of an arm abruptly flailing. Additionally, cross-modality influences, especially an auditory stimulus effecting a visual change, are common.

Phasic us. tonic stimuli: Abrupt, short-lasting (phasic) stimuli and ongoing, longer-lasting (tonic) stimuli appear to affect images in different ways. For images \#1-\#9, phasic influences such as sudden sounds or airplane movements, result in equally abrupt changes in image content, most notably, in people or 


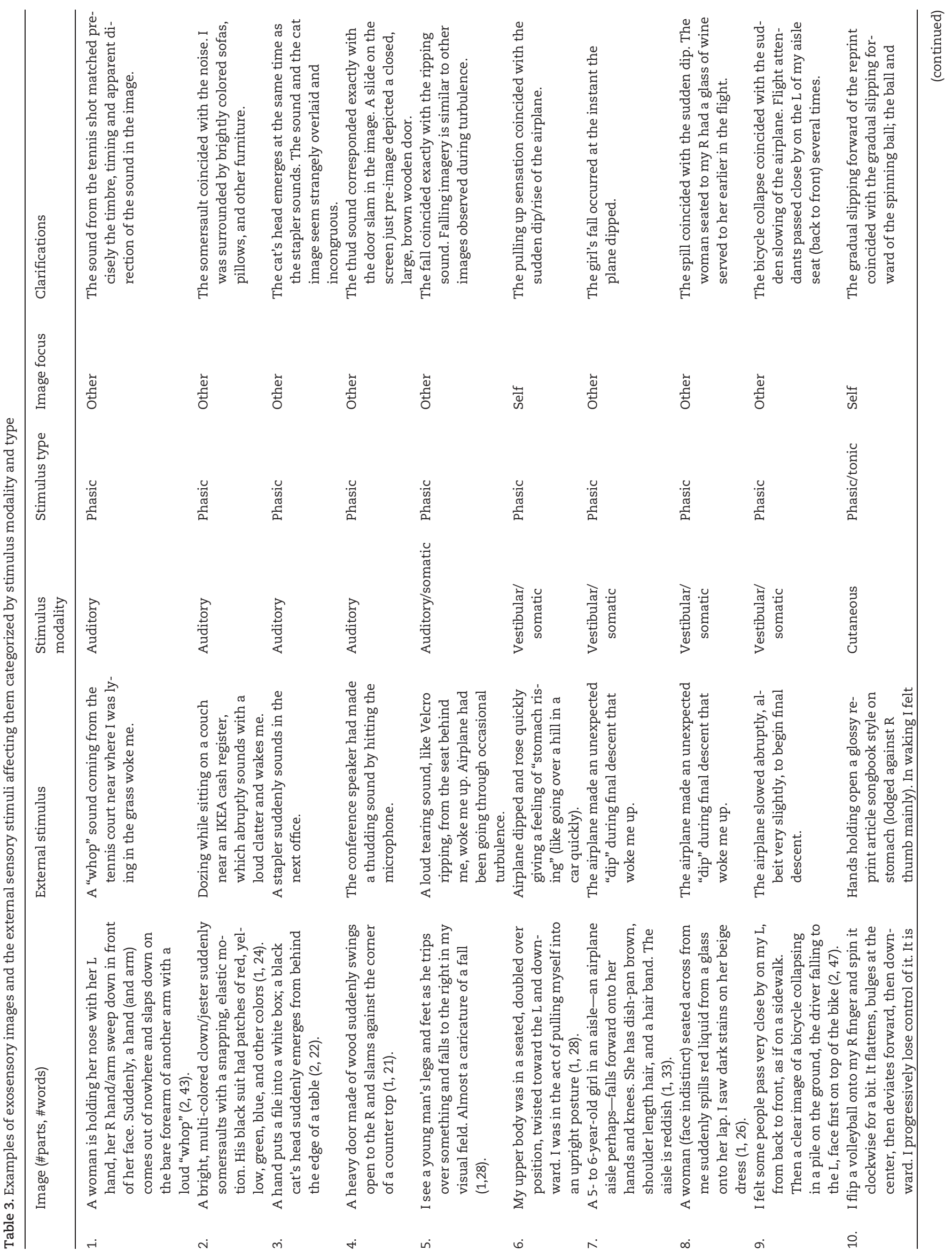




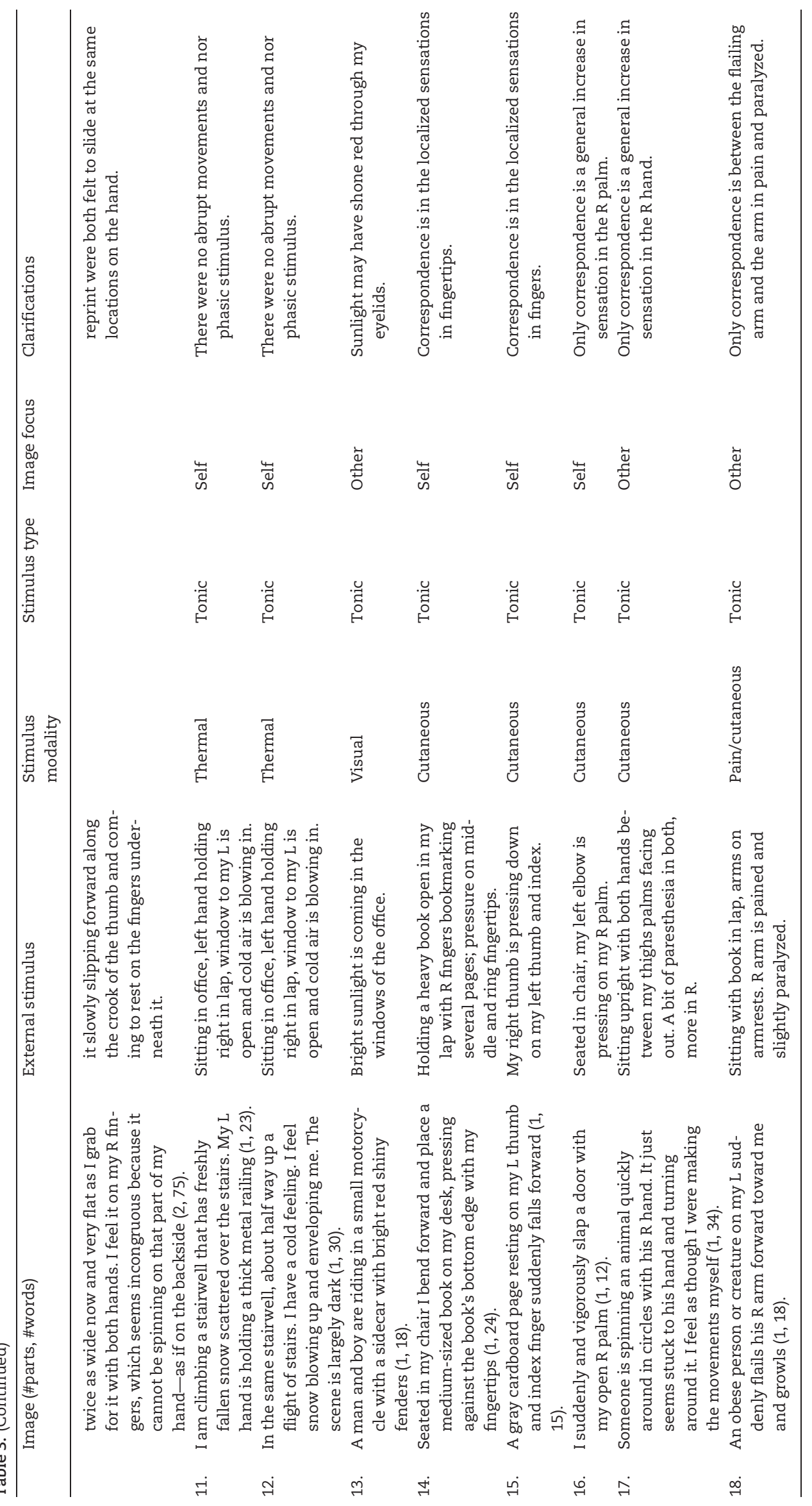


objects falling, appearing quickly, or being hit brusquely. Some changes suggest a direct integration of the stimulus into the image's core, e.g. in image \#1, the dreamed sound of an arm being slapped directly reflects the real sound of a tennis ball being hit; in image \#4, the dreamed sound of a door slamming reflects the real sound of a microphone being struck; and in image \#6, a dreamed sense of pulling the bent-over self into an upright posture reflects the real feeling of rising as an airplane is buffeted abruptly by turbulence.

However, in several other images a phasic stimulus appears to have only indirectly modified an image. In image \#2, an abrupt vestibular stimulus is not incorporated directly as a selffeeling of falling or moving, but as a clown character abruptly somersaulting. Similarly, in images \#7 and \#9 vestibular stimuli lead not to self-feelings of falling, but to other characters falling; in images \#8 and \#9, such stimuli lead to objects falling. Such indirect, "projected" incorporations have also been observed in REM sleep dream imagery after somatosensory stimulation (Dement and Wolpert, 1958; Koulack, 1969; Nielsen, 1993).

Tonic stimuli also sometimes lead to abrupt changes. Images \#15, \#16, and \#18 demonstrate the eruptive quality familiar from phasic stimuli even though in all three cases there was no sudden stimulus. Thus, although there is a general correspondence between both the modality and the phasic or tonic nature of source stimulations, exceptions behoove us to seek explanations beyond a simple mechanism of "direct incorporation" or even "projected incorporation" (see later).

The orderliness of imagery disruption: When disruptions of image formation are induced, the end-content maintains a certain degree of orderliness. The integrity of objects and characters is preserved to varying degrees and the image contents almost inevitably adhere to the physical laws of classical mechanics. For example, images for which stimulation triggered a "falling" theme $(\# 5, \# 7, \# 8, \# 9, \# 10, \# 15)$ all depict objects or characters that fall in a relatively orderly manner, i.e. downwards in accordance with the influence of gravity, not up or sideways as might be expected if no sense of gravity was in play. Further, although some objects in these images do appear to lose their stability within the scene, they do not completely lose their structural integrity. For example, for the spinning ball in image \#10 there is a progressive loss of balance control and round shape, yet the ball changes in a plausible manner ("flattening") rather than bursting or falling apart entirely. Even so, there are again exceptions; in image \#9, a bicycle collapses into a pile, losing most of its structure. Overall, however, the maintenance of an image's stability, even in the face of disruption, points to degrees of resilience of a basic reality simulation process and, more precisely, to relative preservation of the multisensory integration (MSI) of multimodal and self/nonself elements. Even as an image is disrupted there is improvisation of a solution that obeys rules of classical mechanics for the real world. Further, the integration of real perceptual events into imagined ones in this manner suggests that sensory systems may normally be implicated in imagery formation in various ways, a notion that has been proposed both for waking imagery (Finke, 1980; Kosslyn et al., 1995; McNorgan, 2012) and dreaming (Windt, 2015).

\section{Interpretation of causal factors: MSI}

The preceding observations illustrate that sometimes an external stimulus is not only incorporated directly into an image, but it elicits details in other modalities that contextualize this incorporation. This takes place very quickly, possibly within a few hundred milliseconds to, at most, a second or two, and before a full return to consciousness. This rapid elicitation of both direct incorporation and apparent context poses a logical conundrum for an on-line theory of microdream formation, but a conundrum whose resolution might help resolve how more complex dream imagery is so seamlessly improvised. The conundrum hinges on the observation that an external stimulus so quickly elicits a set of image features, some of which seem to temporally precede the stimulus. It is particularly clear in two images from Table 3 that are affected by phasic sounds. For image \#1, the sound of a tennis racquet striking a ball is not only incorporated directly as a hand slap sound, but visual details preceding the slap are also represented, i.e. the hand falling prior to it slapping. For image \#4, the sound of a microphone being bumped is incorporated directly into the image as the sound of a door slamming, yet it also elicits the visual image of a swinging door that seems to precede the sound. The stimuli in both cases presumably elicit-through some associative mechanism-visual details that are appropriate to, and thus help contextualize, the sound stimuli, but that also seem to occur just before the sounds themselves.

One explanation for this type of experiential component reordering is that the external stimulus, an abrupt sound in both examples, is incorporated as a dreamed sound only after a contextualizing visual image has been triggered associatively. There is a precedent for hypothesizing such a selective delay; conscious awareness of a sensory event may not occur until up to $500 \mathrm{~ms}$ after an initial response in sensory cortex; delays are shorter for supra-liminal sensations but longer for nearthreshold events (Libet et al., 1991). Further, individuals are not aware of these delays (Libet et al., 1979). According to Libet (2006): "It is as if this delayed awareness is subjectively referred backwards in time to the time of the primary evoked response of the sensory cortex" (p. 324). According to this view, then, the stimulus sounds in examples \#1 and \#4 may immediately evoke associated visual images, but the sounds and visual images are differentially delayed within the $500-\mathrm{ms}$ window. The sounds may be delayed longer than the visual images or the visual images may be referred backwards in time to a greater extent than are the sounds. In either case, the visual images seem subjectively to occur before the sounds that triggered them.

While the merits of Libet's findings-and especially the limitations of his phenomenological measures-continue to be debated (Lau et al., 2006; Wolpe and Rowe, 2014), their application here is clearly speculative. However, a second, related, approach to dealing with this conundrum and several other features of exosensory images is to consider these images as a special case of MSI (see reviews in De Gelder and Bertelson, 2003; Talsma, 2015). Specifically, exosensory microdreams could be assessed as instances of MSI in which the sensory modalities of an external stimulus and the features of an endogenous image are in conflict (e.g. auditory stimulus vs. visual image). A similar perspective has been supported for interactions between MSI and intentionally generated, waking state, auditory and visual images (Berger and Ehrsson, 2013; Berger and Ehrsson, 2014; Vetter et al., 2014). Microdreams could potentially be studied experimentally with similar methods, e.g. by presenting external stimuli while subjects are falling asleep. These findings for imagery justify us considering how a number of findings from MSI studies may parallel observations of microdreams from the present inquiry. These include findings about: (i) the timing of percepts (For the present discussion, the term "percept" is used to refer to both real sensory information and the representation of such information in either waking or dreaming imagery.) in different sensory modalities, (ii) the perceived localization of these percepts, (iii) the 
perceived intensity of percepts, and (iv) the qualitative alteration of visual percepts by auditory and somatic stimuli.

\section{Percept timing}

During wakefulness, information in different modalities reaches sense receptors at different speeds (e.g. light travels faster than sound) and is processed in sensory systems at different rates. Thus, MSI mechanisms compensate for discrepancies in the timing, localization, and other multisensory features of objects. These mechanisms are thought to overcome intersensory conflicts to preserve the perception of unified objects and, ultimately, a unified stream of consciousness.

In the case of percept timing, evidence indicates that the perceived onset of visual events can be temporally shifted by stimuli in other modalities - but especially by auditory stimuli. Thus, when brief visual and auditory stimuli are presented consecutively, regardless of order, the perceived onset of the visual stimulus shifts toward that of the sound (Fendrich and Corballis, 2001; Bertelson and Aschersleben, 2003; De Gelder and Bertelson, 2003; Morein-Zamir et al., 2003; Jaekl and Harris, 2007). Cutaneous stimulation can also force a time shift of visual events.

If the visual content of microdream imagery behaves like that of visual perception, then visual imagery, too, may be shifted temporally to appear closer in time to an external auditory stimulus. This one mechanism alone may account for how a visual image comes to be perceived prior to the auditory stimulus that triggered it.

\section{Percept localization}

Unlike percept timing, percept localization is more strongly influenced by visual stimuli and may help explain how external stimuli are seamlessly relocalized into microdreams. A sound's apparent location is likely to shift toward that of a visual event if the two occur close enough in time. The visual event is said to "capture" the sound's location as in the "ventriloquist effect" (Howard and Templeton, 1966) in which a visual stimulus (the dummy's mouth) captures the verbal stimulus (the ventriloquist's voice) forcing a multisensory illusion of integration. Such an effect might underlie the production of exosensory imagery once a visual component has been elicited (e.g. images \#2, \#4); the eliciting sound may be captured by the visual image and seem to arise from it rather than from the real world. This is analogous to experiments in which the classic ventriloquist effect occurs when subjects only imagine the visual component of the illusion; an auditory stimulus is drawn toward the visual image's location just as it is for a real visual stimulus (Berger and Ehrsson, 2013). These authors conclude that "mental imagery is capable of integrating with perceptual stimuli of a different sensory modality" (p. 1369). A similar integrative mechanism could explain why incorporation of a sound stimulus into visual microdream imagery usually does not preserve the original location of the stimulus. However, it is noteworthy that such a mechanism may also fail entirely as suggested by image \#1 (Table 3) in which the incorporated sound was perceived to originate exactly from its location in the real world.

\section{Percept intensity}

Sound stimuli may modify the apparent intensity of visual percepts (Stein et al., 1996). For example, if an LED light is presented together with a brief, broad-band auditory stimulus, the light will be rated as more intense than if it is presented aloneespecially when the light is at a low visual intensity. Such auditory stimuli appear to facilitate early stage sensory processing of visual stimuli as suggested by when they e.g. reduce reaction times in visual identification (Doyle and Snowden, 2001) or improve detection of changes in visual motion (Staufenbiel et al., 2011). A similar facilitative mechanism may be implicated in the rapid production of visual imagery at sleep onset when a phasic noise elicits an abrupt facilitation of visual imagery, leading possibly to the "eruptive" quality of elicited images.

\section{Qualitative changes in visual events}

Auditory stimuli can alter visual percepts in several qualitative respects that have implications for image formation processes, including (i) apparent motion and (ii) image segmentation. First, in the "bounce illusion," two small target discs moving in a straight line toward each other, coinciding, then continuing on can be made to seem to "bounce off" each other if a phasic sound burst is presented near the point of coincidence. Even a subliminal sound can induce this effect (Dufour et al., 2008) and the window of coincidence is widened by positive emotion (Kitamura et al., 2015). The pertinence for microdream imagery is demonstrated by the fact that the bounce illusion is induced even when subjects only imagine the phasic sound (Berger and Ehrsson, 2013).

Second, brief sound stimuli can fragment a unitary visual percept into two or more parts. With the sound-induced flash illusion, one phasic light flash accompanied by two rapid beeps will be perceived as two flashes of light; multiple beeps will elicit multiple light flashes (Shams et al., 2002). Multiple tactile stimuli can also elicit this illusion (Violentyev et al., 2005).

These two sets of findings suggest further ways in which exosensory sleep-onset images might be affected by external phasic stimuli. On the one hand, external sounds or tactile sensations may induce "bounce"-like changes in visual imagery such that the latter are induced to change velocity, trajectory, or some other ongoing kinetic feature. On the other hand, phasic sounds might affect the structural integrity of images such that stimulation leads to a fragmentation of imagery in a manner contingent upon stimulus timbre or temporal structure.

\section{MSI approach: synthesis}

These four types of MSI findings applied to exosensory microdream imagery suggest several novel possibilities for explaining basic mechanisms of imagery formation. Findings illustrating MSI changes in percept timing, localization, intensity, and qualitative features such as movement and structural integrity all find ready parallels in the exosensory phenomena identified in microdreams. While there is evidence that MSI principles can be applied to waking imagery formation processes, the present analysis demonstrates at least the proof of concept that their application to microdream imagery is feasible and a potentially fertile avenue for future research and theory building.

\section{References}

Andrillon T, Nir Y, Cirelli C et al. Single-neuron activity and eye movements during human REM sleep and awake vision. Nat Commun 2015;6:1-10.

Arkin AM, Antrobus JS. The effects of external stimuli applied prior to and during sleep on sleep experience. In: Ellman SJ, Antrobus JS (eds), The Mind in Sleep, 2 edn. New York: John Wiley \& Sons, Inc., 1991, 265-307.

Aserinsky E, Kleitman N. Regularly occurring periods of eye motility, and concomitant phenomena during sleep. Science 1953;118:273-74. 
Aspy DJ. Is dream recall underestimated by retrospective measures and enhanced by keeping a logbook? An empirical investigation. Conscious Cogn 2016;42:181-203.

Berger CC, Ehrsson HH. Mental imagery changes multisensory perception. Curr Biol 2013;23:1367-72.

Berger CC, Ehrsson HH. The fusion of mental imagery and sensation in the temporal association cortex. J Neurosci 2014;34:13684-92.

Bertelson P, Aschersleben G. Temporal ventriloquism: crossmodal interaction on the time dimension. 1. Evidence from auditory-visual temporal order judgment. Int J Psychophysiol 2003;50:147-55.

Bertolo H, Paiva T, Pessoa L et al. Visual dream content, graphical representation and EEG alpha activity in congenitally blind subjects. Brain Res Cogn Brain Res 2003;15:277-84.

Bishop C, Rosenthal L, Helmus T et al. The frequency of multiple sleep onset REM periods among subjects with no excessive daytime sleepiness. Sleep 1996;19:727-30.

Brady TF, Konkle T, Alvarez GA et al. Real-world objects are not represented as bound units: independent forgetting of different object details from visual memory. J Exp Psychol Gen 2013;142:791-808.

Chellappa SL, Cajochen C. Ultradian and circadian modulation of dream recall: EEG correlates and age effects. Int $J$ Psychophysiol 2013;89:165-70.

Chellappa SL, Frey S, Knoblauch V et al. Cortical activation patterns herald successful dream recall after NREM and REM sleep. Biol Psychol 2011;87:251-56.

Cicogna P, Cavallero C, Bosinelli M. Cognitive aspects of mental activity during sleep. Am J Psychol 1991;104:413-25.

De Gelder B, Bertelson P. Multisensory integration, perception and ecological validity. Trends Cogn Sci 2003;7:460-67.

Dement W, Kleitman N. Cyclic variations in EEG during sleep and their relation to eye movements, body motility, and dreaming. Electroencephalogr Clin Neurophysiol 1957;9:673-90.

Dement W, Wolpert E. The relationship of eye movements, body motility, and external stimuli to dream content. J Exp Psychol 1958;55:543-53.

Doyle MC, Snowden RJ. Identification of visual stimuli is improved by accompanying auditory stimuli: the role of eye movements and sound location. Perception 2001;30:795-810.

Dufour A, Touzalin P, Moessinger $\mathrm{M}$ et al. Visual motion disambiguation by a subliminal sound. Conscious Cogn 2008;17:790-97.

Esposito MJ, Nielsen TA, Paquette T. Reduced alpha power associated with the recall of mentation from Stage 2 and Stage REM sleep. Psychophysiology 2004;41:288-97.

Fendrich R, Corballis PM. The temporal cross-capture of audition and vision. Percept Psychophys 2001;63:719-25.

Finke RA. Levels of equivalence in imagery and perception. Psychol Rev 1980;87:113-32.

Foulkes D, A Grammar of Dreams. New York: Basic Books, Inc., 1978.

Foulkes D, Dreaming: A Cognitive-Psychological Analysis. Hillsdale, NJ: Lawrence Erlbaum Associates, 1985.

Foulkes D, Fleisher S. Mental activity in relaxed wakefulness. J Abnorm Psychol 1975;84:66-75.

Foulkes D, Scott E. An above-zero baseline for the incidence of momentary hallucinatory mentation. Sleep Res 1973;2:108.

Foulkes D, Vogel G. Mental activity at sleep onset. J Abnorm Psychol 1965;70:231-43.

Freud S, The Interpretation of Dreams. New York: Basic Books, 1900.

Gardner R Jr, Grossman WI, Roffwarg HP et al. The relationship of small limb movements during REM sleep to dreamed limb action. Psychosom Med 1975;37:147-59.

Garrison KA, Scheinost D, Worhunsky PD et al. Real-time fMRI links subjective experience with brain activity during focused attention. Neuroimage 2013;81:110-18.
Germain A, Nielsen TA. EEG power associated with early sleep onset images differing in sensory content. Sleep Res Online 2001;4:83-90.

Hamame CM, Vidal JR, Ossandon T et al. Reading the mind's eye: online detection of visuo-spatial working memory and visual imagery in the inferior temporal lobe. Neuroimage 2012;59:872-79.

Hayashi M, Katoh K, Hori T. Hypnagogic imagery and EEG activity. Percept Mot Skills 1999;88:676-78.

Herman JH, Erman M, Boys R et al. Evidence for a directional correspondence between eye movements and dream imagery in REM sleep. Sleep 1984;7:52-63.

Hohwy J, Bayne T. The neural correlates of consciousness. In: Miller SM (ed.), The Constitution of Phenomenal Consciousness. Toward a Science and Theory. Amsterdam: John Benjamins, 2015, 155-76.

Hori T, Hayashi M, Morikawa T. Topographical EEG changes and the hypnagogic experience. In: Ogilvie R, Harsh JR, eds. Sleep onset: Normal and abnormal processes. Washington, D.C: American Psychological Association, 1994:237-53.

Horikawa T, Tamaki M, Miyawaki Y et al. Neural decoding of visual imagery during sleep. Science 2013;340:639-42.

Howard IP, Templeton WB. Human Spatial Orientation. London: John Wiley \& Sons, 1966.

Hunt H, Ruzycki-Hunt K, Pariak D et al. The relationship between dream bizarreness and imagination: Artifact or essence? Dreaming 1993;3:179-99.

Jaekl PM, Harris LR. Auditory-visual temporal integration measured by shifts in perceived temporal location. Neurosci Lett 2007;417:219-24.

Kerr NH, Domhoff GW. Do the blind literally "see" in their dreams? A critique of a recent claim that they do. Dreaming 2004;14:230-33.

Kerr NH, Foulkes D, Schmidt M. The structure of laboratory dream reports in blind and sighted subjects. J Nerv Ment Dis 1982;170:286-94.

Kitamura MS, Watanabe K, Kitagawa N. Positive emotion facilitates audiovisual binding. Front Integr Neurosci 2015;9:1-9.

Kosslyn SM, Behrmann M, Jeannerod M. The cognitive neuroscience of mental imagery. Neuropsychologia 1995;33:1335-44.

Koulack D. Effects of somatosensory stimulation on dream content. Arch Gen Psychiatry 1969;20:718-25.

Lau HC, Rogers RD, Passingham RE. On measuring the perceived onsets of spontaneous actions. J Neurosci 2006;26:7265-71.

Lehmann D, Dumermuth G, Lange B et al. Dream recall related to EEG spectral power during REM periods. Sleep Res 1981;10:151.

Lehmann D, Faber PL, Achermann P et al. Brain sources of EEG gamma frequency during volitionally meditation-induced, altered states of consciousness, and experience of the self. Psychiatry Res 2001;108:111-21.

Lewis LD, Weiner VS, Mukamel EA et al. Rapid fragmentation of neuronal networks at the onset of propofol-induced unconsciousness. Proc Natl Acad Sci USA 2012;109:E3377-86.

Libet B. Reflections on the interaction of the mind and brain. Prog Neurobiol 2006;78:322-26.

Libet B, Pearl DK, Morledge DE et al. Control of the transition from sensory detection to sensory awareness in man by the duration of a thalamic stimulus. Brain 1991;114:1731-57.

Libet B, Wright EW Jr, Feinstein B et al. Subjective referral of the timing for a conscious sensory experience - a functional role for the somatosensory specific projection system in man. Brain 1979;102:193-224.

Llinas R, Ribary U. Coherent $40-\mathrm{Hz}$ oscillation characterizes dream state in humans. Proc Natl Acad Sci USA 1993;90:2078-81.

Lutz A, Lachaux JP, Martinerie J et al. Guiding the study of brain dynamics by using first-person data: synchrony patterns correlate with ongoing conscious states during a simple visual task. Proc Natl Acad Sci USA 2002;99:1586-91. 
Marzano C, Ferrara M, Mauro F et al. Recalling and forgetting dreams: theta and alpha oscillations during sleep predict subsequent dream recall. J Neurosci 2011;31:6674-83.

Mashour GA. Sleep, anesthesia, and consciousness. Sleep 2011;34:247-48.

Mavromatis A, Richardson JTE. Hypnagogic imagery. In: Sheikh AA (ed.), International Review of Mental Imagery, Vol. 1. New York: Human Sciences Press, 1984, 159-89.

McNorgan C. A meta-analytic review of multisensory imagery identifies the neural correlates of modality-specific and modality-general imagery. Front Hum Neurosci 2012;6:1-14.

Milz P, Faber PL, Lehmann D et al. The functional significance of EEG microstates-Associations with modalities of thinking. Neuroimage 2016;125:643-56.

Morein-Zamir S, Soto-Faraco S, Kingstone A. Auditory capture of vision: examining temporal ventriloquism. Brain Res Cogn Brain Res 2003;17:154-63.

Mouthon AL, Huber R. Methods in pediatric sleep research and sleep medicine. Neuropediatrics 2015;46:159-70.

Nielsen T. Ultradian, circadian, and sleep-dependent features of dreaming. In: Kryger M, Roth T, Dement WC (eds), Principles and Practice of Sleep Medicine, 5th edn. New York: Elsevier, 2011, 576-84.

Nielsen TA. A self-observational study of spontaneous hypnagogic imagery using the upright napping procedure. Imagin Cogn Pers 1992;11:353-66.

Nielsen TA. Changes in the kinesthetic content of dreams following somatosensory stimulation of leg muscles during REM sleep. Dreaming 1993;3:99-113.

Nielsen TA. Describing and modeling hypnagogic imagery using a systematic self-observation procedure. Dreaming 1995;5:75-94.

Nielsen TA. A review of mentation in REM and NREM sleep: 'covert' REM sleep as a possible reconciliation of two opposing models. Behav Brain Sci 2000;23:851-66.

Nielsen TA, Chénier V. Variations in EEG coherence as an index of the affective content of dreams from REM sleep: relationships with face imagery. Brain Cogn 1999;41:200-12.

Nielsen TA, Kuiken D, Alain G et al. Immediate and delayed incorporations of events into dreams: further replication and implications for dream function. J Sleep Res 2004;13:327-36.

Nielsen TA, Powell RA. The day-residue and dream-lag effects: A literature review and limited replication of two temporal effects in dream formation. Dreaming 1992;2:67-77.

Nielsen TA, Stenstrom P. What are the memory sources of dreaming? Nature 2005;437:34-38.

Nieminen JO, Gosseries O, Massimini M et al. Consciousness and cortical responsiveness: a within-state study during non-rapid eye movement sleep. Sci Rep 2016;6:1-10.

Nir Y, Tononi G. Dreaming and the brain: from phenomenology to neurophysiology. Trends Cogn Sci 2010;14:88-100.

Noreika V, Canales-Johnson A, Koh J et al. Intrusions of a drowsy mind: neural markers of phenomenological unpredictability. Front Psychol 2015;6:1-10.

Noreika V, Valli K, Lahtela $\mathrm{H}$ et al. Early-night serial awakenings as a new paradigm for studies on NREM dreaming. Int $J$ Psychophysiol 2009;74:14-18.

Ogilvie RD. The process of falling asleep. Sleep Med Rev 2001;5:247-70.

Oswald I. Sleeping and waking. Physiology and Psychology. Amsterdam: Elsevier, 1962.

Petitmengin C, Lachaux JP. Microcognitive science: bridging experiential and neuronal microdynamics. Front Hum Neurosci 2013;7:1-6.

Poudel GR, Innes CR, Bones PJ et al. Losing the struggle to stay awake: divergent thalamic and cortical activity during microsleeps. Hum Brain Mapp 2014;35:257-69.
Purdon PL, Pierce ET, Mukamel EA et al. Electroencephalogram signatures of loss and recovery of consciousness from propofol. Proc Natl Acad Sci USA 2013;110:E1142-51.

Scarpelli S, D'atri A, Gorgoni M et al. EEG oscillations during sleep and dream recall: state- or trait-like individual differences? Front Psychol 2015;6:1-10.

Schacter DL. The hypnagogic state: a critical review of the literature. Psychol Bull 1976;83:452-81.

Shams L, Kamitani Y, Shimojo S. Visual illusion induced by sound. Brain Res Cogn Brain Res 2002;14:147-52.

Siclari F, Larocque JJ, Postle BR et al. Assessing sleep consciousness within subjects using a serial awakening paradigm. Front Psychol 2013;4:1-9.

Silberer H. Report on a method of eliciting and observing certain symbolic hallucination-phenomena. In: Rapaport D (ed.), Organization and Pathology of Thought. New York: Columbia University Press, 1951, 195-207.

Singh M, Drake CL, Roth T. The prevalence of multiple sleeponset REM periods in a population-based sample. Sleep 2006;29:890-95.

Solms M. The Neuropsychology of Dreams. Mahwah, NJ: Lawrence Erlbaum Associates, 1997.

Staufenbiel SM, van der Lubbe RH, Talsma D. Spatially uninformative sounds increase sensitivity for visual motion change. Exp Brain Res 2011;213:457-64.

Stein BE, London N, Wilkinson LK et al. Enhancement of perceived visual intensity by auditory stimuli: a psychophysical analysis. J Cogn Neurosci 1996;8:497-506.

Stenstrom P, Fox K, Solomonova E et al. Mentation during sleep onset theta bursts in a trained participant: a role for NREM stage 1 sleep in memory processing? Int J Dream Res 2012;5:37-46.

Stickgold R. EMDR: a putative neurobiological mechanism of action. J Clin Psychol 2002;58:61-75.

Talsma D. Predictive coding and multisensory integration: an attentional account of the multisensory mind. Front Integr Neurosci 2015;9:19.

Tanaka H, Hayashi M, Hori T, Coherence analysis of topographical characteristics of the hypnagogic EEG. In: Koga Y, Nagata K, Kirata K (eds), Brain Topography Today. Amsterdam: Elsevier, 1998a, 309-12.

Tanaka H, Hayashi M, Hori T. Topographic mapping of electroencephalography coherence in hypnagogic state. Psychiatry Clin Neurosci 1998b;52:147-8.

Thompson E, Varela FJ. Radical embodiment: neural dynamics and consciousness. Trends Cogn Sci 2001;5:418-25.

Tinguely G, Huber R, Borbely AA et al. Non-rapid eye movement sleep with low muscle tone as a marker of rapid eye movement sleep regulation. BMC Neurosci 2006;7:1-9.

Tirunahari VL, Zaidi SA, Sharma R et al. Microsleep and sleepiness: a comparison of multiple sleep latency test and scoring of microsleep as a diagnostic test for excessive daytime sleepiness. Sleep Med 2003;4:63-67.

van Rijn E, Eichenlaub JB, Lewis PA et al. The dream-lag effect: selective processing of personally significant events during Rapid Eye Movement sleep, but not during Slow Wave Sleep. Neurobiol Learn Mem 2015;122:98-109.

Verdone P. Temporal reference of manifest dream content. Percept Mot Skills 1965;20:1253-68.

Vetter P, Smith FW, Muckli L. Decoding sound and imagery content in early visual cortex. Curr Biol 2014;24:1256-62.

Violentyev A, Shimojo S, Shams L. Touch-induced visual illusion. Neuroreport 2005;16:1107-10. 
Vogel GW. Sleep-onset mentation. In: Ellman SJ, Antrobus JS (eds), The Mind in Sleep: Psychology and Psychophysiology, 2nd edn. New York, NY: John Wiley \& Sons, 1991, 125-42.

Wamsley EJ, Perry K, Djonlagic I et al. Cognitive replay of visuomotor learning at sleep onset: temporal dynamics and relationship to task performance. Sleep 2010;33:59-68.

Werth E, Achermann P, Borbely AA. Selective REM sleep deprivation during daytime. II. Muscle atonia in non-REM sleep. Am J Physiol Regul Integr Comp Physiol 2002;283: R527-32.

Windt JM. The immersive spatiotemporal hallucination model of dreaming. Phenomenol Cogn Sci 2010;9:295-316.

Windt JM, Dreaming: A Conceptual Framework for Philosophy of Mind and Empirical Research. Cambridge, MA: MIT Press, 2015.

Wolpe N, Rowe JB. Beyond the urge to move: objective measures for the study of agency in the post-Libet era. Front Hum Neurosci 2014;8:1-13. 